\title{
Multilinguales
}

\section{Intéractions verbales des enseignants d'anglais en réunion pédagogique à l'université Constantine I}

Teachers of English verbal interactions in pedagogical meetings at Constantine 1 University

\section{Amina Amel Azil}

\section{CpenEdition}

\section{Journals}

Édition électronique

URL : http://journals.openedition.org/multilinguales/1673

DOI : 10.4000 /multilinguales. 1673

ISSN : 2335-1853

\section{Éditeur}

Université Abderrahmane Mira - Bejaia

\section{Édition imprimée}

Date de publication : 1 juin 2014

Pagination : 201-213

ISSN : 2335-1535

\section{Référence électronique}

Amina Amel Azil, «Intéractions verbales des enseignants d'anglais en réunion pédagogique à l'université Constantine I », Multilinguales [En ligne], 3 | 2014, mis en ligne le 03 juin 2014, consulté le 17 septembre 2019. URL : http://journals.openedition.org/multilinguales/1673 ; DOI : 10.4000/ multilinguales. 1673

Ce document a été généré automatiquement le 17 septembre 2019.

\section{(1) $\$$}

Multilinguales est mise à disposition selon les termes de la Licence Creative Commons Attribution Pas d'Utilisation Commerciale - Pas de Modification 4.0 International 


\title{
Intéractions verbales des enseignants d'anglais en réunion pédagogique à l'université Constantine I
}

\author{
Teachers of English verbal interactions in pedagogical meetings at Constantine 1 \\ University
}

Amina Amel Azil

1 Notre étude ne s'intéresse pas à l'interaction verbale entre les enseignants et leurs apprenants en salle de cours, mais plutôt aux interactions verbales entre enseignants d'une même langue, pendant les réunions pédagogiques conçues comme situation de communication verbale bien déterminée.

2 Le choix de la réunion dans notre recherche se justifie par le fait qu'elle est, de notre point de vue, la situation de communication la plus appropriée parce qu'elle nous permet d'observer, dans un cadre cohérent, les interactions verbales de membres d'une seule catégorie professionnelle, les enseignants d'anglais, réunis par des objectifs communs de nature pédagogique. Si, comme le précise V. Traverso (1999), «l'interaction correspond à ce qui se passe lorsque plusieurs personnes se trouvent réunies » (p. 5), le cadre de la réunion pédagogique la rend encore plus pertinente pour l'objet de notre recherche. La réunion est, en effet, une tâche pédagogique officielle et une situation de travail formelle où les actes de communication se produisent et où différents messages sont échangés. Ces actes de communication sont déterminés par l'ordre du jour de la réunion, les objectifs visés, les règles de travail bien définies ainsi que des différents rôles et statuts des interlocuteurs. Selon Kerbrat-Orecchioni (1998) :

«Tout au long du déroulement d'un échange communicatif quelconque, des différents participants, que l'on dira donc des "intéractants", exercent les uns sur les autres un réseau d'influences mutuelles - parler, c'est échanger, et c'est changer en échangeant. » (p.17) 
3 Ainsi, toutes ces conditions de l'échange en réunion influencent les productions linguistiques des participants. Mais ce qui a retenu notre attention au préalable c'était de constater qu'au département d'anglais de l'université de Constantine I, l'environnement linguistique, jusqu'à l'affichage pédagogique, était dominé par l'usage d'autres langues que l'anglais. Ce constat a suscité une série de questions sur les langues utilisées par les enseignants d'anglais eux-mêmes lorsqu'ils communiquent entre eux : dans leurs différentes réunions de travail, l'anglais est-il la seule langue utilisée dans leurs interactions verbales ? S'ils recourent à plusieurs langues, quel est le rôle de chacune d'elles?

4 Notre hypothèse est que la langue anglaise, langue d'enseignement et d'apprentissage au département d'anglais, n'est pas la langue dominante des interactions verbales des enseignants de cette langue durant leurs réunions de travail.

\section{Démarche méthodologique}

5 Nous allons analyser les interactions verbales des enseignants durant ces réunions de travail afin de cerner les phénomènes linguistiques qui les caractérisent, d'expliciter les raisons du recours à d'autres langues que l'anglais, et de déterminer le rôle de chacune des langues utilisées.

6 Notre démarche méthodologique a consisté en des enregistrements d'un certain nombre de discours interactifs oraux des enseignants, que nous avons transcrits orthographiquement et phonétiquement, et cela en adoptant les conventions de transcription élaborées par Traverso (1999). Nous avons adopté ce modèle de transcription en fonction des éléments constitutifs de notre corpus enregistré.

7 La méthode d'enquête que nous avons suivie, pour l'enregistrement de ces interactions verbales, est l'observation non-participante afin que le discours interactif des enseignants ne soit pas perturbé par notre intrusion et que les données recueillies soient authentiques. Nous nous sommes présentée auprès de ces enseignants en tant qu'étudiante de master en didactique de langue, préparant un mémoire sur la coordination pédagogique des enseignants. Il est à noter que ces enregistrements ont été effectués dans la salle réservée aux réunions et durant le mois de janvier 2012.

Le corpus recueilli contient les interactions de trois réunions pédagogiques d'une durée de cinq (5) heures. Celles-ci auraient dû être analysées intégralement, mais elles représentent un corpus d'un volume dont l'examen nécessite plus d'espace que nous n'en disposons pour cette contribution. Notre analyse s'est portée donc uniquement sur une seule réunion, d'une heure et trente minutes et se compose de vingt-huit séquences. Son ordre du jour est l'élaboration, par les enseignants du module d'expression écrite de troisième année de licence (L3), du sujet d'examen de la fin du premier semestre. Participent à cette réunion l'enseignant responsable du module et animateur de la réunion, et deux enseignantes de la matière.

9 A l'enregistrement de ces interactions verbales, nous avons ajouté les réponses à un questionnaire que nous avons conçu comme un outil de recherche complémentaire afin de collecter des données supplémentaires sur nos sujets informateurs et sur leurs collègues d'anglais. Notre but est de donner plus de crédit à notre analyse et à nos conclusions. Pour l'élaboration du questionnaire, nous avons adopté la méthode des quotas : il a été destiné à tous les enseignants d'anglais toutes catégories confondues, y 
compris ceux et celles qui ont participé aux réunions pédagogiques. Nous n'avons retenu aucun critère de sélection pour que le corpus des réponses soit le plus représentatif possible.

Ce questionnaire comprend deux parties: la première porte sur le profil des enseignants d'anglais (âge, sexe, diplôme, grade et nombre d'années enseignées à l'université), et la deuxième sur les langues qu'ils emploient dans différentes situations de communication. L'objectif du questionnaire est de cerner les raisons qui poussent ces enseignants à utiliser telle ou telle langue pendant leurs différentes réunions pédagogiques.

\section{Les formes de l'alternance codique}

Les données recueillies ont révélé que les enseignants d'anglais utilisaient bien souvent et d'une manière concomitante quatre langues : le français, l'arabe dialectal, l'anglais et l'arabe scolaire. En effet, en examinant de près le discours interactif des enseignants, nous avons constaté que pour mieux établir le contact entre eux et pour mieux faire passer leurs messages, ces enseignants recouraient au français, à l'arabe dialectal, à l'anglais et à l'arabe scolaire. Ils les utilisent pour donner des explications et apporter plus de précisions sur les différentes lacunes de leurs apprenants en expression écrite, et aussi sur le choix ainsi que sur l'élaboration des sujets d'examen. Il s'agit donc, pour eux, de faire de l'alternance codique le premier instrument pour assurer une communication efficace, fondée sur la juxtaposition linguistique de ces quatre langues.

Voici un échantillon des formes d'alternances codiques enregistrées.

\section{Alternances codiques intra-phrastiques}

Dans cet exemple (Enseignant1) s'adresse à (Enseignante2) qui vient d'arriver pour lui faire un compte rendu du début de la réunion :

1E. 1 : (s'adressant à Enseignant2) Juste euh pour euh noter $\uparrow[$ bərək]

// // ce que nous avions dit tout à l'heure donc euh Madame :: [X]

elle m'a appelé euh [qatli] euh j'ai des empêchements $\uparrow$ // [an:: i::] euh je vais :: vous envoyer par e-mail les sujets finalement elle n'a pas envoyé son sujet euh j'ai vérifié euh j'ai bien vérifié :: :: tout à l'heure [fə] la bibliothèque centrale // // elle n'a pas envoyé [jaßni] que dire euh pour dire que [ऊna] on est $::::$ là on travaille on fait le sujet ensemble on collabore :::::::: mais ////////dans le cas où quelqu'un est absent donc euh euh si on fait notre sujet on va // // $/ /$ on n'impose pas finalement parce que les gens [ma3awə]] :: $\uparrow$ (inaudible) voilà.

2 E.T.2 :oui.

3 E. 1 : voilà donc [hdərT ana ou] mademoiselle $[\mathrm{X}] \downarrow$ tout à fait.

14 Au niveau du premier tour de parole (1E.1), l'alternance codique est intra-phrastique entre le français et l'arabe dialectal. Nous remarquons, ici, que le français est la langue dominante. L'arabe dialectal est utilisé pour l'adverbe de liaison [bərək]= "seulement », le verbe introducteur [qatli] = « elle m'a dit », le segment [an :: $i]$ = «je suis ", l'indicateur de lieu $[f \ni]=$ «dans » qui renvoie au mot "la bibliothèque ", 
l'indicateur de reformulation [jaSni ] = "c'est-à-dire ", le pronom personnel [§na ]$=$ ] nous » et l'expression «les gens [ $\mathrm{ma3aw} / \mathrm{J}]$ » = « les gens ne viennent pas ».

Dans cette expression, l'alternance est intra-phrastique parce que les éléments caractéristiques des langues en présence sont utilisés dans un rapport syntaxique très étroit :

- le sujet est en français : les gens

- le verbe, par contre, est en arabe dialectal : [ma3awəf] = « ne viennent pas ». Dans le dernier tour de parole (3E.1), l'alternance codique est intra-phrastique de type prédicatcomplément. Le recours à l'arabe dialectal est constaté dans l'utilisation du verbe [hdərt] = «j'ai parlé ».

En observant attentivement ce passage, nous constatons que la construction syntaxique [hdərt ana ou] mademoiselle [X] offre les caractéristiques internes des deux langues en présence :

(hderT ana ou) (mademoiselle $\mathrm{X})=$ «j'ai parlé avec mademoiselle $\mathrm{X}$ »

\begin{tabular}{|l|l|l|}
\hline$\downarrow$ & $\downarrow$ & (traduction littérale) \\
\hline (prédicat) & (complément) & \\
\hline en & en & \\
\hline arabe dialectal & français & \\
\hline
\end{tabular}

\section{Alternances codiques inter-phrastiques}

5E. 1: ah euh une minute [bərk] // est ce que vous pouvez le lire pour moi s'il vous plait ok euh bon $\uparrow$ c'est bien should we punish different cheaters in the same way.

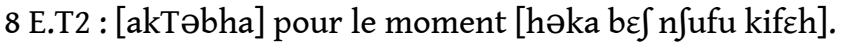

11 E. 1 : non :: c'est juste entre parenthèses on va discuter ça euh c'est tout c'est tout [haka bərk] on ouvre euh on ferme la parenthèse.

Une alternance codique inter-phrastique entre l'anglais et le français est relevée au niveau du cinquième tour de parole (5E.1). L'anglais est utilisé pour la formulation du sujet d'examen : should we punish different cheaters in the same way $=$ « devrions-nous punir deux étudiants fraudeurs de la même manière ». Le français est par contre repéré au niveau des segments suivants : le nom « une minute » utilisé ici pour exprimer une demande, l'adjectif «bon » et l'expression « c'est bien » exprimant l'approbation.

Dans le huitième tour de parole (8E.T.2), nous avons une alternance inter-phrastique. Le seul segment alterné en langue française est l'indicateur de temps «pour le moment » qui sert à suggérer une proposition provisoire du sujet d'examen.

L'alternance codique présente dans le onzième tour de parole (11E.1) est une alternance codique inter-phrastique entre le français et l'arabe dialectal. Le segment alterné est: [haka bərk] =« c'est bien comme ça ? » sert à attirer l'attention des deux enseignantes.

1E. 1 : (il regarde enseignante 3) [ana] il me semble :: $\uparrow::[$ mənaSraf] :: $\uparrow$

c'est pour un petit peu // // parce que notre objectif ce n'est pas de :: de euh de voir 
s'ils ont euh suffisamment d'informations sur le sujet lui-même $\uparrow$ des informations bon [mlis] c'est bien c'est </>.

2E.T.2: [hih].

3E.T.3 : oui c'est vrai.

21 Au niveau du premier tour de parole (1E.1), nous remarquons une alternance codique inter-phrastique entre le français et l'arabe dialectal. Le recours à l'arabe dialectal est repéré au niveau des segments suivants : l'indicateur de personne [ana] =« moi » qui sert à établir la distance entre enseignant et apprenants, le verbe $[$ məna $\mathrm{S} r a f]=\ll$ je ne sais pas » et l'adverbe [mli $]$ ] = « c'est bien » qui a la fonction d'un régulateur.

Notons qu'entre le deuxième tour de parole (2E.T.1) et le troisième (3E.T.3), il y a une alternance codique inter-phrastique entre le français et l'arabe dialectal. Le segment alterné en arabe dialectal est l'adverbe d'affirmation [hih] = « oui ».

\section{Alternance codique extra-phrastique}

\section{E.1 :Bonjour//bon euh [bismi leh araЂmen eraЋim] euh bon on commence.}

niveau de ce tour de parole, l'alternance codique entre le français et l'arabe dialectal est extra-phrastique. L'arabe dialectal est utilisé au niveau de la formule religieuse [bismi leh araЋmen eraЂim] =«au nom de Dieu le Tout Miséricordieux, le Très Miséricordieux " (traduction littérale), à travers laquelle est affirmée l'appartenance religieuse des enseignants.

Il est à noter que la forme d'alternance codique la plus fréquente, dans le discours interactif de ces enseignants, est l'alternance intra-phrastique. Nous pensons que cela est dû à la nature des segments alternés qui s'insèrent dans la structure interne des différents systèmes grammaticaux en présence : adverbes, verbes, pronoms personnels, connecteurs et conjonctions de coordination en arabe dialectal ou termes techniques relevant de l'enseignement de l'expression écrite en français ou en anglais.

Le plus souvent cette alternance codique intra-phrastique est du type prédicatcomplément : (prédicat en arabe, complément en français). L'usage récurrent de cette forme d'alternance codique montre que les enseignants ont une compétence linguistique bilingue quasi-équilibrée.

La forme d'alternance codique la moins constatée dans notre corpus est l'alternance codique inter-phrastique. Quant à l'alternance codique extra-phrastique, elle n'est notée qu'une seule fois, au début de l'interaction, par l'usage de la formule religieuse évoquée plus haut. Sur le plan linéaire, nous avons constaté que les formes d'alternance codique variaient au niveau des tours de parole. Certains passages contiennent deux formes d'alternance, les alternances intra-phrastique et inter-phrastique, alors que d'autres n'ont qu'une seule forme d'alternance, l'alternance intra-phrastique ou l'alternance inter-phrastique.

\section{Les fonctions de l'alternance codique}

Dans notre corpus, l'alternance codique accomplit les fonctions suivantes :

- la personnalisation du message, qui montre l'implication des locuteurs dans leurs propos : elle se manifeste, presque dans toutes les productions linguistiques des enseignants, par leur 
usage des pronoms personnels : $[a n a]=$ "moi », [Ћna] = «nous », par des indicateurs de possession : $[t \varepsilon\lceil n a]=$ «notre », [bənəsba lia $]=$ «pour moi », ou par des prédicats comme $[\mathrm{\Upsilon}$ andna $]=\ll$ nous avons $» ;$

- la réitération : elle est utilisée par les locuteurs pour renforcer la communication, s'assurer de la bonne interprétation des messages, et aider à la compréhension des idées. [bənəsba lia] = « pour moi », [ Gandna $]=$ « nous avons »;

- la citation : elle sert aux locuteurs à rapporter les propos d'autrui ; elle est, en général, initiée en arabe dialectal par l'utilisation des verbes introducteurs [qultəlhum] = « je leur ai dit », [qali] = « il m'a dit », et achevée en français ;

- la désignation d'un interlocuteur : elle se fait généralement par le biais d'un terme d'adresse (le prénom de l'interlocuteur ou un terme de respect) précédé de la particule vocative [a] ; nous avons également noté une autre fonction de l'alternance codique : la désignation d'un interlocuteur absent par l'usage du pronom personnel [huma] = «ils » qui renvoie aux apprenants ;

- la modalisation d'un message et la reformulation : pour préciser ou modaliser le contenu de leur discours, les locuteurs utilisent les indicateurs de reformulation [jaßni] = «c'est-àdire,$[$ səma $]=$ « autrement dit »;

- le récit ou la narration : elle est constatée lorsque les enseignants énumèrent ce qu'ils ont fait en salle de cours avec leurs apprenants ;

- la hiérarchie des statuts ou distance entre enseignants et apprenants : elle se manifeste par l'utilisation des pronoms personnels [§na] = « nous » et [huma] =

- «ils», par les interlocuteurs pour établir la distance hiérarchique entre enseignants et apprenants ;

- le mixage de codes : il est effectué par le transfert d'éléments linguistiques de la langue arabe vers les langues française et anglaise : au niveau des verbes par l'adjonction des affixes de l'arabe dialectal à la racine des verbes français, [nkominikiw] = «nous communiquons », [nmotiviouhum] = «nous les motivons ", au niveau des substantifs par l'adjonction du déterminant arabe « $\mathrm{el}$ » ou « 1 » aux mots français, « el'paragraphe » ou anglais «l'spelling $»=$ « l'orthographe ».

\section{Le rôle des langues dans la réunion de travail} des enseignants, nous pouvons d'abord dire que la langue française est une langue véhiculaire; elle sert, bien souvent, à faire circuler l'information entre les interlocuteurs. D'après notre étude, cette langue est utilisée pour les termes techniques appartenant au domaine de l'enseignement du module de l'expression écrite: " orthographe", "ponctuation", "grammaire», etc. Les interactants recourent également à la langue française pour se saluer (bonjour), pour remercier (merci), pour s'adresser la parole avec civilité (monsieur, madame), pour réguler le déroulement de l'interaction par l'usage des indicateurs d'approbation (oui, voilà). Néanmoins, les énoncés en langue française sont truffés d'éléments verbaux en arabe (dialectal ou scolaire) et en anglais.

S'agissant de l'arabe dialectal, les locuteurs y recourent pour assurer l'enchaînement des différentes parties de leur discours grâce notamment aux embrayeurs [wəla] = «ou », [bəli] = «que », [li] = « qui », aux démonstratifs [hədi] = «cette », [hada huwa] = 
"c'est ça ", aux indicateurs de personnes [ana] = "moi », [huma] = «ils", et aux indicateurs de formulation [jaini] = « c'est-à-dire », [səma] = « autrement dit ».

Par ailleurs, l'arabe dialectal est utilisé pour signifier des traits culturels et religieux. Citons la formule de salutation [asalemu Salajkum] = «que la paix soit sur vous », celle du serment $[$ wəlahi $]=$ "je jure par Allah », l'interjection $[n f a l a h]=$ "si Dieu le veut », celle consacrée à toute entrée en matière [bismi leh araЂmen eraЂim] = « traduction ».

L'arabe dialectal est également doté d'une fonction expressive. Les enseignants l'utilisent pour exprimer leur satisfaction : [ja؟rfu jaktbu] = «ils savent rédiger », [†ə3 buni] = «ils m'ont plu », leur mécontentement [məjməduf fəl wəkt be $\left.\int j ə k t b u\right]=\ll$ ils ne prennent pas suffisamment de temps pour rédiger un essai ", [məSndhum $\int$ məjəIrfu jəkTbuh] = «ils n'ont pas l'essai expositif, ils ne savent pas le rédiger ». Ils expliquent ces défaillances des apprenants au niveau de leurs productions écrites par le recours à des méthodes d'enseignement qu'ils jugent inadaptées et par un volume horaire d'enseignement du module très réduit : [saSa ou nos mahiS kafia] = « une heure et demi, ce n'est pas suffisant ».

Notons enfin que notre étude a montré que l'arabe dialectal est utilisé aussi pour exprimer des actions par des prédicats: $[x d ə m t]=$ «j'ai enseigné », [dərna $]=$ «nous avons fait ", pour réguler l'interaction par l'usage des indicateurs d'approbation [hih] = «oui », pour contester une idée et la rectifier par l'usage de l'indicateur de négation $[a h a]=\ll$ non $»$.

34 Concernant l'usage de l'anglais dans les interactions verbales des enseignants en réunions pédagogiques, l'examen de notre corpus a révélé que les locuteurs recouraient à la langue anglaise quand il s'agissait de termes techniques appartenant au domaine de l'enseignement de l'expression écrite: «spelling» = "l'orthographe», "refutation paragraph» = "paragraphe contre argument», "argumentative essay»= "l'essai argumentatif », la formulation du sujet « should the Algerian government engage in building nuclear power plants?» = « est-ce que le gouvernement algérien devrait s'engager dans la construction des centrales nucléaires?", pour l'illustration des fautes des apprenants [hour] = « heure ».

Nous avons constaté un seul cas où l'anglais est utilisé pour la citation directe: l'enseignant rappelle ce qu'il avait dit à ses apprenants en salle de cours : « je leur ai dit que " they are going euh you're going to fail at the end, write clear position that you defend " = «ils vont, vous allez échouer à la fin; écrivez un point de vue clair que vous allez défendre ».

Nous avons relevé un seul cas où cette langue est utilisée par nécessité; en atteste l'hésitation de l'interactant : " all the ways » = « tous les moyens ».

Quant à l'arabe scolaire, d'après nos observations, il se limite à la citation des exemples par l'usage du segment [maقalən] = "par exemple». Nous avons également relevé un cas où l'arabe scolaire servait à introduire la possession par l'usage du segment [bənə sba lia] = « pour moi ».

38 Ainsi, l'analyse des interactions verbales des interlocuteurs a révélé que :

- dans l'ensemble des énoncés, le français est la langue dominante ;

- l'arabe dialectal se limite à l'utilisation des éléments linguistiques tels que les embrayeurs : $[$ wəla $]=$ « ou », [bəli $]=$ « que ». Leur usage fait partie des habitudes langagières des locuteurs (l'utilisation récurrente); 
- dans le cas où la matrice du discours est en arabe dialectal, les langues française et anglaise sont réservées à des termes techniques.

\section{Langues utilisées par les enseignants en contextes interactifs}

\section{Langues utilisées en salles de cours}

A la question «Quelle(s) langue(s) utilisez-vous dans vos salles de cours ? a) Français b) Arabe dialectal $c$ ) Anglais d) Arabe scolaire ", sur les 100 enseignants interrogés, 80 ont affirmé qu'ils utilisaient l'anglais et 20 qu'ils introduisaient du français.

\section{Langues utilisées entre collègues}

A la question «Quelle(s) langue(s) utilisez-vous avec vos collègues enseignants? a) Français b) Arabe dialectal c) Anglais d) Arabe scolaire", sur les 100 enseignants interrogés, 95 disent s'exprimer souvent en français et en arabe dialectal, 5 dans trois langues, le français, l'arabe dialectal et l'anglais.

\section{Langues utilisées en réunions de travail}

41 A la question «Quelle(s) langue(s) utilisez-vous en réunions de travail pédagogique ? a) Français $b$ ) Arabe dialectal c) Anglais $d$ ) Arabe scolaire ", les réponses coïncidaient avec les résultats des interactions verbales en réunion. Sur les 100 enseignants, 80 ont déclaré recourir au français, les 20 autres ont déclaré recourir au français et parfois à l'arabe dialectal. Mais tous les enseignants réservent l'anglais aux cours (en situation pédagogique). D'après toutes ces réponses, nous pouvons dire que la langue anglaise est sous-utilisée par les enseignants d'anglais.

\section{Les représentations des langues française et anglaise}

A la question "dans vos réunions pédagogiques, préférez-vous utiliser le français? ", sur les 100 enseignants, 95 ont répondu par l'affirmative.

Les raisons qu'ils ont avancées sont :

- le recours à la langue française serait automatique,

- en réunion pédagogique, la langue française serait un outil de communication efficace : les enseignants d'anglais préfèreraient utiliser le français parce qu'ils communiqueraient mieux en réunions de travail. Les 5 autres enseignants, qui ont répondu négativement, n'ont donné aucune justification.

45 A la question «comparativement à l'anglais, le français est-il une langue facile ou difficile?", sur les 100 enseignants, 98 la trouvent «facile " parce que, d'après eux, le français est la première langue étudiée et parlée, après la langue maternelle (arabe dialectal, berbère); ils l'utilisent plus facilement que ce soit au travail, ou avec leurs amis, etc. 


\section{BIBLIOGRAPHIE}

KERBRAT-ORECCHIONI C., Les interactions verbales, approche interactionnelle et structure des conversations, Paris, Armand Colin, 1998.

TRAVERSO V., L'analyse des conversations, Paris, Nathan, 1999.

\section{ANNEXES}

\section{Annexe I : Les conventions de transcription (Traverso, 1999)}

Les mots inaudibles sont indiqués par : (inaudible).

Les phrases inachevées sont indiquées par : (inachevée). Les hésitations : euh

Les comportements non verbaux sont indiqués entre parenthèses. Les interruptions par un autre interactant $:</>$

Les séquences traduites sont mises entre guillemets. Les pauses : // // // (selon la longueur de la pause)

Allongements vocaliques ::: ou :::: ou :: :: :: (Selon la longueur). Transcription phonétique : [ ]

Les marques d'intonation :

$\uparrow$ : intonation montante.

$\downarrow$ : intonation descendante. 
! : Intonation exclamative.

Dans notre transcription du corpus, les locuteurs sont désignés par (E.1) pour l'Enseignant1, (E.T.2) pour l'Enseignant2 et (E.T.3) pour l'Enseignante 3. Les personnes dont ils parlent sont désignées par les lettres $\mathrm{X}, \mathrm{Y}$.

Les nombres sont notés en chiffres lorsqu'ils sont en langue française, transcrits orthographiquement lorsqu'ils sont en langue anglaise et transcrits phonétiquement lorsqu' ils sont en arabe.

Les langues française et anglaise ayant des lettres latines sont transcrites orthographiquement. Par contre, les séquences en arabe sont transcrites phonétiquement selon les symboles proposés par l'API.

Annexe II : Le tableau de l'ensemble des symboles (Haddid, 2007)

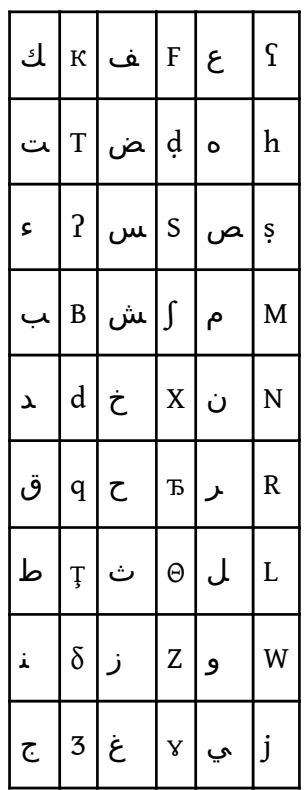

*Pour la transcription phonétique du son (ث), spécifique au dialecte constantinois, nous avons gardé le symbole (q) qui désigne le son (ق).

Les voyelles : $\varepsilon$ : ouvert. - ə : fermé

\section{RÉSUMÉS}

Notre étude s'intéresse aux interactions verbales des enseignants du département d'anglais, université Constantine I. Après avoir enregistré leurs discours interactifs en réunions pédagogiques, et utilisé un questionnaire comme outil de recherche complémentaire, l'analyse de notre corpus a montré que les pratiques langagières des enseignants cités sont caractérisées par l'alternance du français, de l'arabe dialectal/scolaire et de l'anglais. Un des principaux résultats de cette étude est que le français est la langue dominante dans les interactions verbales de ces enseignants, dans leur espace linguistique supposé être anglophone, où l'anglais est la langue d'enseignement et d'apprentissage. 
Our study focuses on the verbal interactions of university teachers at the department of English, Constantine University I. After recording some of their interactive discourses in pedagogical meetings, we used a questionnaire as a complementary research tool. The analysis of our corpus revealed that these teachers' language practices are characterized by code switching from French to classical/Algerian Arabic/ and English. One of the major findings in this study is that French is the dominant language used by teachers of English in their oral interactions in a supposed English speaking environment where the medium of teaching and learning is the English language.

\section{INDEX}

Mots-clés : interactions verbales, pratiques langagières, alternance codique, réunions pédagogiques, enseignants d'anglais

Keywords : verbal interaction, language practices, code switching, pedagogical meetings, teachers of English

\section{AUTEUR}

\section{AMINA AMEL AZIL}

Université Constantine I - Algérie

AZIL Amina Amel prépare une thèse de doctorat en sciences du langage, à l'Université Constantine 1. 